\title{
Wavefront Reconstruction from Slope Data Within Pupils of Arbitrary Shapes Using Iterative Fourier Transform
}

\author{
Guang-Ming Dai*
}

AMO Laser Vision Correction Group, 3400 Central Expressways, Santa Clara, CA 95051, USA

\begin{abstract}
This letter presents a new modal technique that reconstructs a wavefront using iterative Fourier transforms and converts the Fourier coefficients as needed to and from the coefficients of the orthonormal basis set over pupils of arbitrary shapes. It is shown that, for wavefront slope data, modal reconstruction with Fourier series using an iterative algorithm is faster and more accurate than when an orthonormal basis set is used. Elliptical, annular, hexagonal, and irregular pupils are considered as practical examples to illustrate the results.
\end{abstract}

\section{INTRODUCTION}

Wavefront reconstruction from wavefront slope data has been discussed at length in the literature [1-6]. Zernike polynomials are used with the singular value decomposition (SVD) algorithm [6] when the pupil is circular. For noncircular pupils, however, an orthonormal set over that pupil must be used [7].

This Letter extends the application of Fourier series [8] to wavefront reconstruction over pupils of arbitrary shapes. The relationships between the Fourier coefficients and the coefficients of an orthonormal basis set are derived. Modal reconstruction of wavefronts from slope data is demonstrated using iterative Fourier transforms. Because of its natural tie to the Nyquist limit, the Fourier technique utilizes the discrete wavefront-slope information optimally, i.e., using the spatial frequency information exactly up to the Nyquist limit. In the SVD technique using the orthonormal basis set, however, reconstructing too few modes results in a large residual error [6]. Therefore, the optimal number of modes is automatically determined in Fourier technique but varies depending on the sample size over which the wavefront is to be reconstructed in the SVD technique. Also the fast Fourier transform makes wavefront reconstruction using the iterative Fourier technique faster than the SVD algorithm. Examples of noncircular pupils such as elliptical, annular, and hexagonal are given to cover practical applications of eyes with elliptical pupils [9] and telescopes with central obscuration [10] or with hexagonal mirrors [11]. An irregular pupil is also included in the study.

\section{THEORY}

Consider a wavefront defined by a domain $S$ in Cartesian coordinates, denoted as $W(\boldsymbol{x})$. Suppose a complete and analytical set of basis functions $\left\{Z_{i}(\boldsymbol{x})\right\}$ is orthonormal over domain $S$; we can expand the wavefront into this set as

$W(\mathbf{x})=\sum_{i=1}^{L} c_{i} Z_{i}(\mathbf{x})$

*Address correspondence to this author at the AMO Laser Vision Correction Group, 3400 Central Expressway, Santa Clara, CA 95051, USA;

E-mail: George.Dai@amo-inc.com where $L$ is the total number of expansion terms and the expansion coefficient $c_{i}$ can be calculated from the orthonormality as

$c_{i}=\iint P_{S}(\mathbf{x}) W(\mathbf{x}) Z_{i}(\mathbf{x}) \mathrm{d} \mathbf{x}$

where $\mathrm{d} \boldsymbol{x}=\mathrm{d} x \mathrm{~d} y$ and $P_{S}(\boldsymbol{x})$ is the domain function bounded by $S$. The integration in Eq. (2) and elsewhere in this Letter covers the infinite plane. If $S$ is a circular pupil, then $\left\{Z_{i}(\boldsymbol{x})\right\}$ is the set of Zernike polynomials.

Suppose domain $T$ is inscribed in domain $S$ such that $T \in S$. If the wavefront is only known within domain $T$, Eq. (2) can no longer be used because the basis set $\left\{Z_{i}(\boldsymbol{x})\right\}$ is not orthogonal over domain $T$. Instead, a set of orthonormal basis function $\left\{F_{i}(\boldsymbol{x})\right\}$ can be determined with the GramSchmidt orthogonalization process as [7]

$F_{i}(\mathbf{x})=\sum_{l=1}^{L} M_{i l} Z_{l}(\mathbf{x})$

where $M_{i l}$ is the conversion matrix that can be calculated recursively. Therefore

$W(\mathbf{x})=\sum_{i=1}^{L} b_{i} F_{i}(\mathbf{x})$

Once the set of $\left\{F_{i}(\boldsymbol{x})\right\}$ is determined, we can obtain the expansion coefficients over domain $T$ by use of the orthnormality as

$b_{i}=\iint P_{T}(\mathbf{x}) W(\mathbf{x}) F_{i}(\mathbf{x}) \mathrm{d} \mathbf{x}$

where $P_{T}(\boldsymbol{x})$ is the domain function bounded by $T$.

Now, suppose we want to reconstruct a wavefront from its slope data over the domain T. In this Letter, the ANSI standard for Zernike polynomials [12] was used as the basis set $\left\{Z_{i}(\boldsymbol{x})\right\}$. One way to reconstruct the wavefront from slope data is to use the SVD algorithm over the discrete data with the orthonormal basis set $\left\{F_{i}(\boldsymbol{x})\right\}$ within domain $\mathrm{T}$, as demonstrated by Dai [6].

We do this by the use of the iterative Fourier transform method $[5,8]$. Define domain $P$ as a rectangular area inscribing domain $\mathrm{T}$, or $T \in P$. Using sinusoidal functions (Fourier 
series) as the basis set, we can write the wavefront expansion as

$W(\mathbf{x})=\iint a(\mathbf{u}) \exp (j 2 \pi \mathbf{u} \cdot \mathbf{x}) \mathrm{d} \mathbf{u}$

where $j^{2}=-1, \mathrm{~d} \boldsymbol{u}=\mathrm{d} u \mathrm{~d} v$, and $a(\boldsymbol{u})$ is the matrix of the expansion, or the Fourier coefficients (Fourier spectrum). These coefficients are given by

$a(\mathbf{u})=\iint W(\mathbf{x}) \exp (-j 2 \pi \mathbf{u} \cdot \mathbf{x}) \mathrm{d} \mathbf{u}$

Substituting Eq. (6) into (5), we get

$b_{i}=\iint a(\mathbf{u}) V_{i}^{*}(\mathbf{u}) \mathrm{d} \mathbf{u}$

where * stands for the complex conjugate, and the Fourier transform of $\left\{F_{i}(\boldsymbol{x})\right\}$

$V_{i}(\mathbf{u})=\iint P_{T}(\mathbf{x}) F_{i}(\mathbf{x}) \exp (-j 2 \pi \mathbf{u} \cdot \mathbf{x}) \mathrm{d} \mathbf{x}$

can be calculated either analytical or numerically. Substituting Eq. (4) into Eq. (7), and considering that $\left\{F_{i}(\boldsymbol{x})\right\}$ is only supported within the domain $T$, we obtain

$a(\mathbf{u})=\sum_{i=1}^{L} b_{i} V_{i}(\mathbf{u})$

Note that Eqs. (8) and (10) are exact only when $F_{i}(\boldsymbol{x})$ is analytical or the wavefront is sampled at least two times the highest spatial frequency of the wavefront.

Taking derivatives to $x$ and to $y$ on both sides of Eq. (6) and noticing the fact that the derivative of Fourier series is also Fourier series, we can obtain [8]

$a(\mathbf{u})=-j \frac{u b_{u}(\mathbf{u})+v b_{v}(\mathbf{u})}{2 \pi|\mathbf{u}|^{2}}$

where $b_{u}(\boldsymbol{u})$ and $b_{v}(\boldsymbol{u})$ are the Fourier transforms of the slope data. With Eq. (11), the estimated wavefront can be calculated with Eq. (6).

Equation (11) applies only to un-bounded functions, i.e., when $W(\boldsymbol{x})$ does not have a boundary. However, the wavefront to be reconstructed is bounded by domain $\mathrm{T}$, the boundary condition of the slope data must be considered, which lead to the iterative algorithm [5]. Without this iterative algorithm, the reconstructed wavefront near the periphery of the domain boundary is in general underestimated.

\section{RESULTS}

To demonstrate the effectiveness of the Fourier technique, common pupils of elliptical, annular, hexagonal, and irregular shape were used. The aspect ratio of the elliptical pupil is 0.85 , and the obscuration ratio of the annular pupil is 0.35 . A noise-free random wavefront was generated with the first four orders of Zernike polynomials. Wavefront slope data was calculated within these four different pupils using sampling size of $N=401 \times 401$. Both the SVD technique (with reconstruction of the first four orders) and the Fourier technique were used to reconstruct the wavefronts. With the Fourier technique, the first four orders of coefficients of the
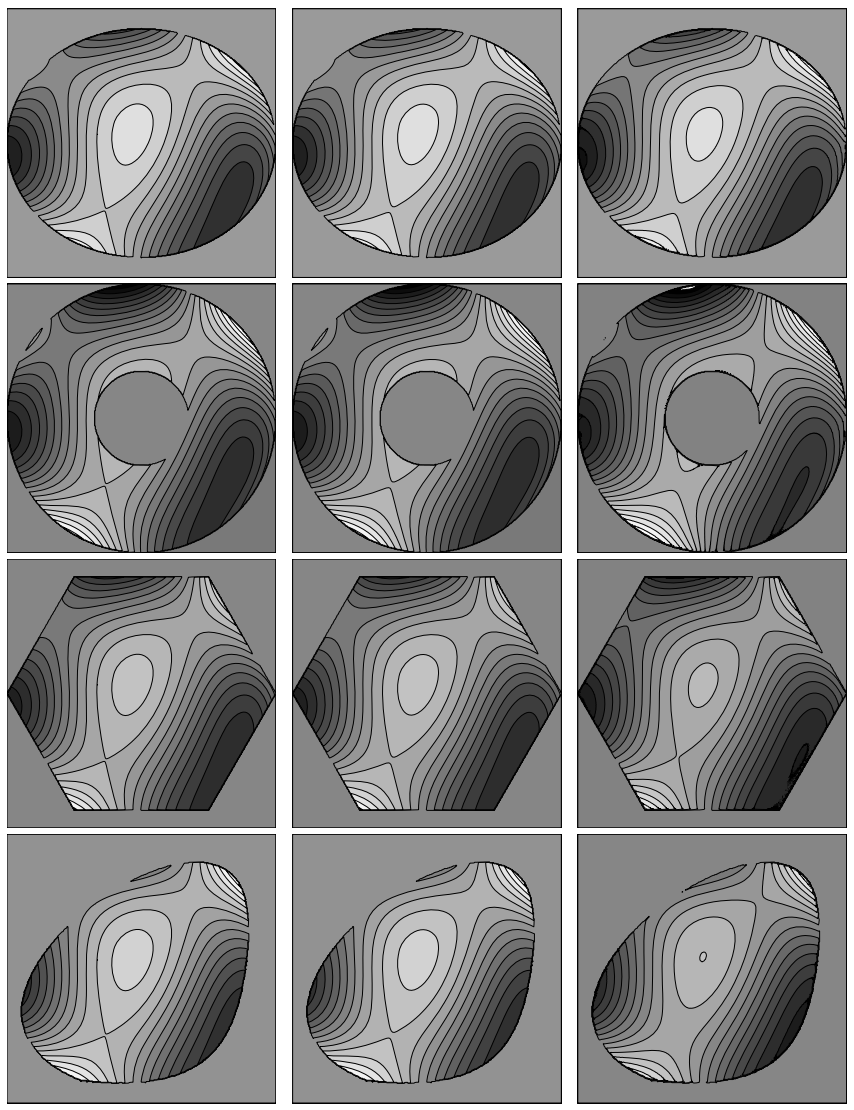

Fig. (1). Contour plots for the input (first column), SVDreconstructed (middle column), and Fourier-reconstructed (last column) wavefronts. Four Zernike orders were used for the input wavefronts. The contour scales for all plots are identical.

basis set $\left\{F_{i}(\boldsymbol{x})\right\}$ were calculated using Eq. (8), and 20 iterations were used for each case. The Fourier transforms of $\left\{F_{i}(\boldsymbol{x})\right\}$ for these pupils were calculated using FFT.

\section{Table 1. RMS Error $(\mu \mathrm{m})$ in the Reconstruction}

\begin{tabular}{|c|c|c|}
\hline Aperture & SVD & Fourier \\
\hline \hline Elliptical & 0.001 & 0.117 \\
\hline Annular & 0.002 & 0.096 \\
\hline Hexagonal & 0.001 & 0.036 \\
\hline Irregular & 0.001 & 0.250 \\
\hline
\end{tabular}

Fig. (1) depicts the input, the SVD-reconstructed, and the Fourier-reconstructed wavefronts for the elliptical, annular, hexagonal, and irregular pupils. The corresponding root mean square (RMS) error is shown in Table 1. The SVDreconstructed wavefronts show very good agreement with the input wavefronts, and so do the coefficients. Fourier technique also shows its effectiveness, but with inferior results. Unfortunately, in a real situation, the number of terms in the reconstruction is always smaller than that in the unknown input wavefront to prevent aliasing. 

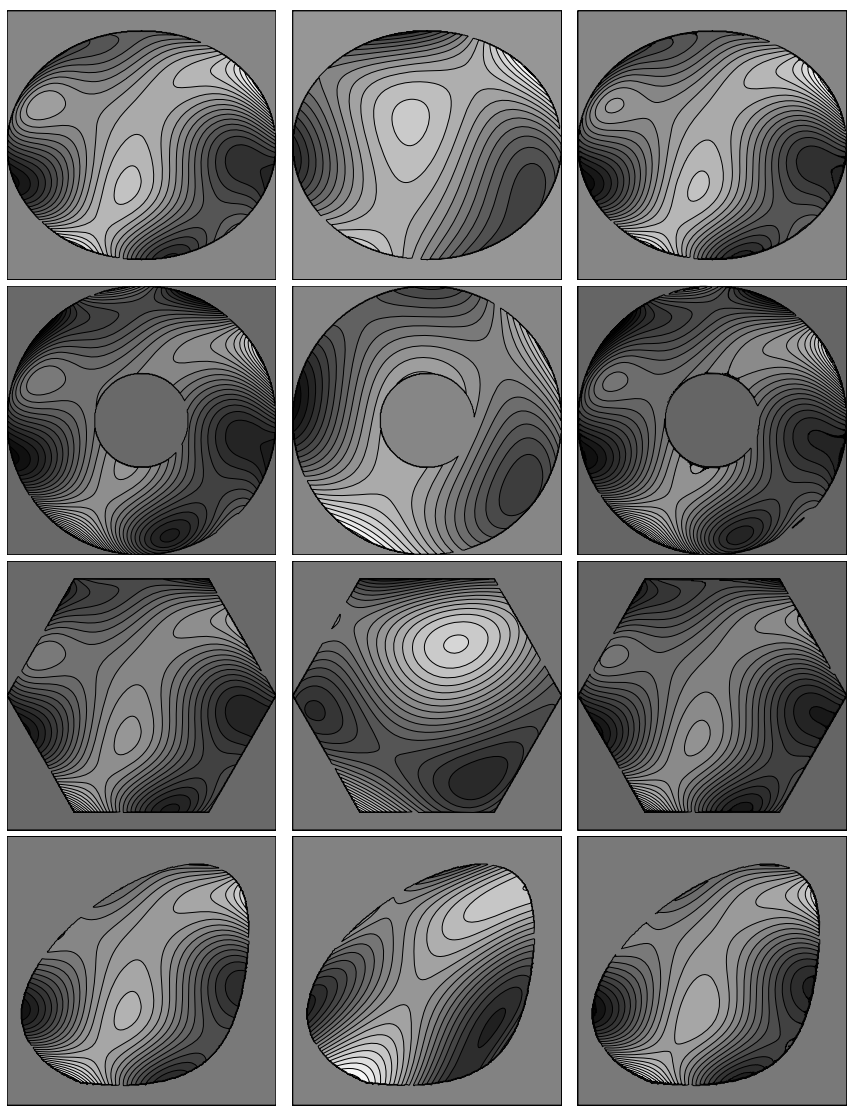

Fig. (2). Contour plots for the input (first column), SVDreconstructed (middle column), and Fourier-reconstructed (last column) wavefronts. Six Zernike orders were used for the input wavefronts. The contour scales for all plots are identical.

Table 2. RMS Error $(\mu \mathrm{m})$ in the Reconstruction

\begin{tabular}{|c|c|c|}
\hline Aperture & SVD & Fourier \\
\hline \hline Elliptical & 0.202 & 0.048 \\
\hline Annular & 0.357 & 0.095 \\
\hline Hexagonal & 0.676 & 0.044 \\
\hline Irregular & 0.348 & 0.171 \\
\hline
\end{tabular}

Six Zernike orders were used in the input wavefront.

When the number of terms in the input wavefront was increased from four Zernike orders to six, the performance of the Fourier technique was superior to the SVD technique as the Fourier technique uses the information from the discrete data optimally. Fig. (2) shows the input and reconstructed wavefronts for these four different pupils. The similarity between the first column and the last column in Fig. (2) indi- cates the benefit of using iterative Fourier technique than the SVD technique. The corresponding RMS error is shown in Table 2. From the RMS error it is clear that the Fourier technique works better than the SVD technique. Finally Table $\mathbf{3}$ shows the reconstruction time for the two techniques for different sample sizes. The Fourier technique is faster than the SVD technique.

Table 3. Comparison of the Wavefront Reconstruction Time (Seconds) for Different Sampling Sizes

\begin{tabular}{|c|c|c|}
\hline $\mathbf{N}$ & SVD & Fourier \\
\hline \hline 100 & 0.016 & 0.000 \\
\hline 400 & 0.031 & 0.002 \\
\hline 1600 & 0.063 & 0.016 \\
\hline 10000 & 0.453 & 0.141 \\
\hline 40000 & 1.781 & 0.609 \\
\hline
\end{tabular}

\section{CONCLUSIONS}

In this Letter, we have shown that the iterative Fourier reconstruction is faster and more accurate in practical cases for wavefront reconstruction from slope data for pupils of arbitrary shapes.

\section{REFERENCES}

[1] Fried DL. Least-square fitting a wave-front distortion estimate to an array of phase-difference measurements. J Opt Soc Am 1977; 67: $370-374$

[2] Southwell WH. Wave-front estimation from wave-front slope measurements. J Opt Soc Am 1980; 70: 998-1005.

[3] Wallner EP. Optimal wave-front correction using slope measurements. J Opt Soc Am 1983; 73: 1771-1776.

[4] Freischlad KR, Koliopoulos CL. Modal estimation of a wave front from difference measurements using the discrete Fourier transform. J Opt Soc Am 1986; A 3: 1857-1861.

[5] Roddier F, Roddier C. Wavefront reconstruction using iterative Fourier transforms. Appl Opt 1991; 30: 1325-1327.

[6] Dai G.-M. Modal wave-front reconstruction with Zernike polynomials and Karhunen-Loève functions. J Opt Soc Am 1996; A 13: 1218-1225.

[7] Mahajan VN. Dai G.-m. Orthonormal polynomials for hexagonal pupils. Opt Lett 2006; 31: 2462-2464.

[8] Dai G.-m. Zernike aberration coefficients transformed to and from Fourier series coefficients for wavefront representation. Opt Lett 2006; 31: 501-503.

[9] Wyatt HJ. The form of the human pupil. Vis Res 1995; 35: 2021 2036.

[10] Scharmer GB. Brown DS. Pettersson L. Rehn J. Concepts for the Swedish 50-cm vacuum solar telescope. Appl Opt 1985; 24: 25582564.

[11] Sabatker E. Burge J. Sabatker D. Analytic diffraction analysis of a 32-m telescope with hexagonal segments for high-contrast imaging. Appl Opt 2005; 44: 1360-1365.

[12] Z80.28-2004, Methods for reporting optical aberrations of eyes, American National Standard Institute (2004). 